\title{
BMJ Open Organised screening for cervical cancer in France: a cost-effectiveness assessment
}

\author{
Stéphanie Barré, ${ }^{1}$ Marc Massetti, ${ }^{2}$ Henri Leleu, ${ }^{2}$ Frédéric De Bels ${ }^{1}$
}

To cite: Barré S, Massetti M, Leleu $\mathrm{H}$, et al. Organised screening for cervical cancer in France: a cost-effectiveness assessment. BMJ Open 2017;7:e014626. doi:10.1136/ bmjopen-2016-014626

- Prepublication history and additional material for this paper are available online. To view please visit the journal (http:// dx.doi.org/10.1136/bmjopen2016-014626).

Received 17 November 2016 Revised 16 August 2017 Accepted 21 August 2017

CrossMark

${ }^{1}$ Screening Department, French National Cancer Institute (INCa), Boulogne-Billancourt, île-deFrance, France

${ }^{2}$ Modeling \& Simulation, Public Health Expertise, Paris, France

Correspondence to

Stéphanie Barré;

sbarre@institutcancer.fr

\section{ABSTRACT}

Objective According to the third cancer plan, organised screening (OS) of cervical cancer (CC) among women aged 25-65 years should be implemented in France in the forthcoming years. The most efficient way to implement OS in the French healthcare system is yet to be determined.

Methods A microsimulation model was developed adopting a collective 'all payers' perspective. A closed cohort of women eligible for CC screening and representative in terms of age and participation in individual screening (IndScr) by annual Papanicolaou (Pap) testing every 3 years was modelled on a lifetime horizon. Different OS strategies, additive to IndScr with a $61.9 \%$ participation rate based on mailed invitations to nonparticipant women to perform OS were assessed. Similar modalities were applied to OS and IndScr participants. Strategies implied different screening tests (Papanicolaou (Pap) test, human papillomavirus (HPV) test and p16/Ki67 double staining) and OS periodicity.

Results Compared with IndScr only, all OS strategies were associated with decreased cancer incidence/ mortality (from $14.2 \% / 13.5 \%$ to $22.9 \% / 25.8 \%$ ). Most strategies generated extra costs ranging from $€ 37.9$ to $€ 1607$ per eligible woman. HPV testing every 10 and 5 years were cost saving. HPV tests every 10 and 5 years were the most efficient strategies, generating more survival at lower costs than Pap-based strategies. Compared to IndScr only, an HPV test every 10 years was cost saving. The most effective strategies were p16/ Ki67 as primary or HPV positive confirmation tests, with respective incremental cost-effectiveness ratios of $€ 6$ 541250 and $€ 101391$ per life year. Pap-based strategies generated intermediary results.

Conclusion OS strategies based on the HPV test appear highly efficient. However, our results rely on the assumption that women and practitioners comply with the recommended $0 S$ periodicities $(3,5,10$ years). Implementing these OS modalities will require major adaptations to the current CC screening organisation. Pap test-based strategies might be simpler to setup while preparing an appropriate implementation of more efficient OS screening modalities.

\section{BACKGROUND}

The natural history of cervical cancer (CC) is related to a persistent human papillomavirus (HPV) infection of the cervix leading

\section{Strengths and limitations of this study}

A microsimulation model was developed to assess the efficiency of possible cervical cancer organised screening strategies in France.

- The model operates on individual women who are eligible for screening and representative of the current French population on a lifetime horizon.

- Real-life practices and data were used, allowing for the fine modelling of the screening and validation against observed data.

- The lack of precision of transition probabilities in the context of a low incidence of cervical cancer and the assumptions required to model screening practices after primary human papillomavirus tests are the main limitations of the study.

to squamous intraepithelial lesions that can evolve into cancerous lesions. CC prevention is based on screening to detect and remove lesions at the early stages to prevent invasive cancer and an anti-HPV vaccination to reduce cancer-associated HPV infection. ${ }^{1}$

In France, CC prevention is based on individual voluntary screening (IndScr) for CC of women aged 25-65 years and vaccination. IndScr is based on a Papanicolaou test (Pap test) every 3 years, after two annual Pap tests that are negative. Approximately, 90\% of Pap smears are done by gynaecologists, although general practitioners and midwives are also authorised to perform it. IndScr has led to a significant decrease in the incidence and associated mortality of CC in the past 20 years. In 2012, CC was the 11th most frequent and 12 th most lethal form of cancer in women. ${ }^{2}$ However, many women still do not participate in CC screening. Participation in IndScr was found to be approximately $61 \%$ of eligible women, with low access to healthcare, comorbidities and poverty being risk factors for non-participation.

Screening remains the main prevention tool in France, as anti-HPV vaccination is restricted to younger age groups and was only recently made available. Furthermore, 
Table 1 Strategies compared

\begin{tabular}{llll}
\hline Strategy & IR+ improved follow-up & Primary test & $\begin{array}{l}\text { Confirmation test after } \\
\text { positive primary test }\end{array}$ \\
\hline Current & No & Pap test/3years & Pap test or HPV \\
Pap/Pap & Yes & Pap test/3years & Pap test \\
\hline Pap/p16Ki67 & Yes & Pap test/3years & p16/Ki67 \\
HPV/Pap-5y* & Yes & HPV/5years & Pap test \\
\hline HPV/Pap-3y* & Yes & HPV/3years & Pap test \\
\hline HPV/Pap-10y* & Yes & HPV/10years & Pap test \\
HPV/p16Ki67-5y* & Yes & HPV/5years & p16/Ki67 \\
HPV/p16Ki67-10y* & Yes & HPV/10years & p16/Ki67 \\
p16Ki67/p16Ki67 & Yes & HPV/3years & p16/Ki67 \\
\hline
\end{tabular}

*Women aged 25-35 are not eligible for HPV screening and receive a Pap test every 3 years instead. Women who tested HPV+/confirmationgo through double testing (HPV+Pap) the following year.

HPV, human papillomavirus; IndScr, individual screening; IR, invitation+recall for woman who did not participate in IndScr in the last 3 years (non-participant); OS, organised screening; Pap, Papanicolaou.

vaccination has had a slow adoption in the French population. In 2015, it was estimated that only $17 \%$ of women eligible for vaccination were vaccinated (Institut de Veille Sanitaire. Vaccination rate among women under 30, personal communication, 2015). ${ }^{3}$ In 2014, the third French Cancer Plan has been presented to address both the human and the societal challenges of cancer. CC organised screening (OS) implementation among women aged 25-65 years is part of its first operational objective and aims at a participation rate of $80 \%$ and a $30 \%$ reduction in CC-related mortality by $2019 .{ }^{4}$

Several OS experimentations have been performed in France to assess the efficacy of different screening modalities, including invitation and positive tests follow-up (FU), self-sampling and HPV testing. Experimentations that consisted of an invitation of non-participants to perform a Pap test allowed to catch up with $13.2 \%$ of all eligible women after 3 years and reduced the lost to follow-up (LtFU) rates of women after a positive result. ${ }^{5}$ Additionally, primary HPV testing and self-sampling were shown to be a feasible alternative to the Pap smear in France. ${ }^{6} 7$ Finally, innovative testing, such as p16/Ki67 double staining, was shown to be a performant alternative for CC screening compared with HPV screening or the Pap test. ${ }^{8}$

Consequently, many alternative strategies can be considered for the implementation of OS for CC in France. Thus, a medico-economic evaluation of several OS strategies based on a cost-effectiveness analysis was performed by the French national cancer institute (INCa), which relied on a scientific steering committee that involved clinical experts and stakeholder representatives (social security, ministry of health, patients and professionals) providing advice on the methodological choices and best OS implementation modality in the French context.

In order to assist decision-making regarding the implementation of CC OS, our study's main outcomes correspond to the objectives of CC OS implementation: participation rate, survival and avoided CC. A cost-utility analysis was performed as well.

\section{METHODS}

Seven strategies were compared with the current IndScronly situation (table 1). These strategies were all based on adding to the current IndScr with the dispatch of screening invitations (followed by a single recall) to women who did not spontaneously participate in the last 3 years (non-participants). Hence, women who did not participate in regular screening are the only ones targeted by the interventions. OS strategies also included improved FU, resulting in a reduction in $\mathrm{LtFU}$ women.

Different screening tests were considered for primary screening or confirmation after a positive primary test, including Pap test, HPV DNA detection and p16/Ki67 double staining. The women who tested positive for both primary and confirmation tests went through colposcopy and conisation if a high-grade (grade 2 or worse cervical intraepithelial neoplasia (CIN) 2+) CIN lesion was identified. Women with CIN1 were retested at 12, 18 and 24 months if the initial lesion was atypical squamous cells of undetermined significance or low-grade squamous intraepithelial lesion on Pap or went through colposcopy. The women who tested positive for a primary test and negative for confirmation were retested after 1 year. A fraction of the participants was $\mathrm{LtFU}$. Women could only be invited once per cycle. Detailed screening algorithms are available in the online supplementary file 1.

The population was limited to women aged 25-65 years who are currently eligible for IndScr.

\section{Model structure}

Given the complexity of screening algorithms (different testing/retesting frequencies) and interactions between participation rates and individual characteristics (age and social), a Markov state microsimulation model was 


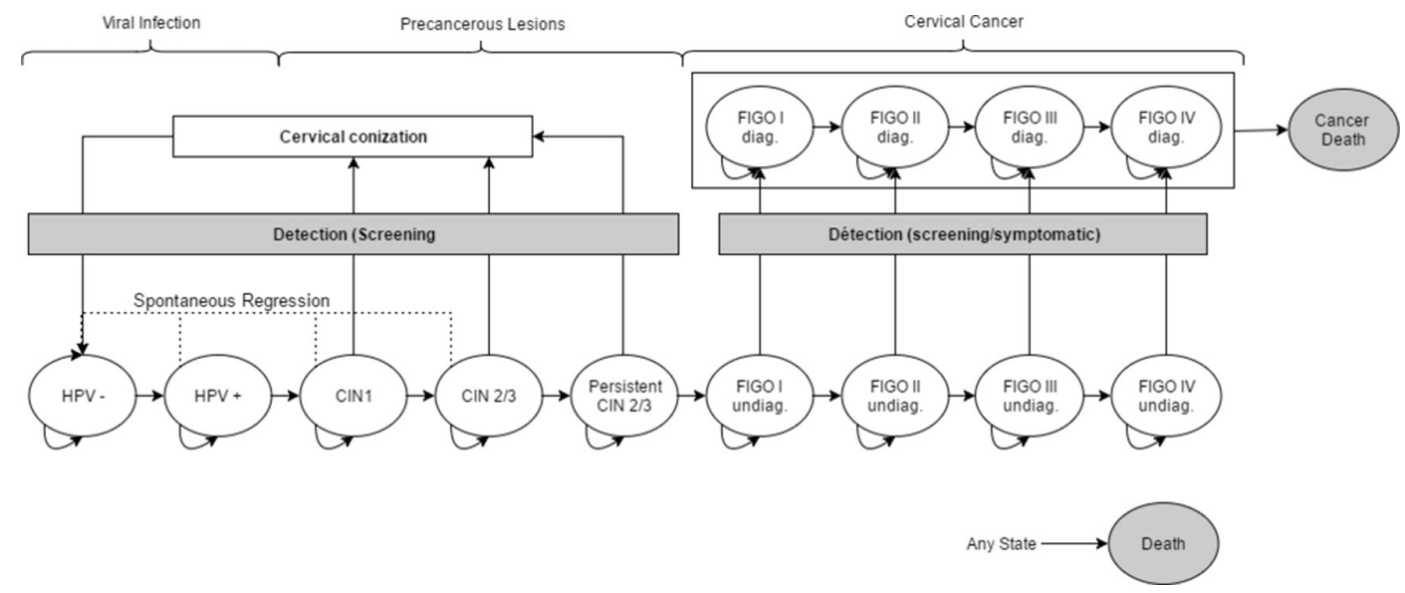

Figure 1 Structure of the model for the natural history of cervical cancer.

developed. Considering the relatively slow progression of intraepithelial lesions and the long-term benefits of screening, a 1-year cycle length was used. The model was adapted from a previously published cohort-based Markov model. ${ }^{9}$ A cohort of 100000 women was simulated. Due to the long-term development of the disease and its consequences, a lifetime horizon was applied.

The model first generates a woman with a randomly attributed age, IndScr participation and frequency, health state (HPV-, HPV+, CIN lesions or cancer) and vaccination attributes. At each cycle, women can progress through states that correspond to CC natural history: non-infected women can get an HPV infection according to an age-dependent and vaccination-dependent risk. The infection can progress to CIN1, then CIN2/3 and finally Federation of Gynaecology and Obstetrics (FIGO)-1 classified non-invasive cancer. HPV infection and CIN lesions can regress spontaneously until CIN2/3 lesions have become persistent (pCIN2/3). Women in the pCIN2/3 state systematically progress to cancer at an age-dependant rate. FIGO I lesions can progress to FIGO II, III and IV and become symptomatic. Once symptomatic, the lesion is treated and the woman remains in the corresponding treated state with an associated cancer mortality rate. An age-specific general mortality applies at any state.

Each year, the model determines whether the woman undergoes screening individually or after invitation based on her participation periodicity, time since last screening and participation rates after invitation. Invitations are sent to non-participant women in the manner of prevention campaigns, following the screening recommended frequency (ie, 3 years in the case of Pap/Pap). Therefore, only a fraction of non-participant women are invited every year. The same primary screening modality is applied to OS and IndScr participants. Screening test results (positivity and lesion type for Pap tests and positivity for HPV and p16/Ki67) are determined based on the current state and type of test performed (see online supplementary file 2). After diagnosis, women with a non-cancerous lesion return to the non-infected state after conisation and cancerous lesions are treated. The structure of the modelled natural history is presented in figure 1 . More details on the model structure are given in the online supplementary file 3 .

\section{Input data}

The input data used in the simulation are presented in table 2.

The population characteristics are based on available epidemiological and demographic data that are representative of the French population. Vaccination status is only determined in women $\leq 30$ years old, as it was only recently available in France. IndScr participation and frequency depend on age and social status and based on the national health insurance database (see online supplementary file 4 ), approximately $61.9 \%$ of eligible women were found to participate in IndScr at a frequency $\leq 4$ years. ${ }^{3}$ Distribution of each modelled health state by age was not available in France and was estimated by simulating a cohort of non-vaccinated 14-year-old women undergoing current IndScr-only screening over their lifetime (see online supplementary file 3).

Transition probabilities (TP) were based on a previously published model. ${ }^{9}$ The HPV infection and pCIN2/3 to cancer progression probabilities were calibrated using the model to reproduce observed HPV and cancer prevalence by age. ${ }^{10}$ The high-risk HPV annual infection rate was estimated to be $3.5 \%-14 \%$, depending on age. ${ }^{11}$ The impact of vaccination is simulated by applying a relative risk (RR) of infection. ${ }^{1}$

Probabilities of cancer progression and emergence of symptoms were obtained from the CC natural history simulation model developed by Myers et al. ${ }^{12}$ The cancer specific-mortality by grade and time since diagnosis was estimated from the data of the american Surveillance, Epidemiology, and End Results programusing data for white women under 50 , as it was assumed that non-specific mortality was low in this group. ${ }^{13}$ General mortality was modelled according to the French national statistics office (INSEE) data.

The participation rates after invitation and recall, $\mathrm{LtFU}$ rate associated with IndScr, OS effect on LtFU 


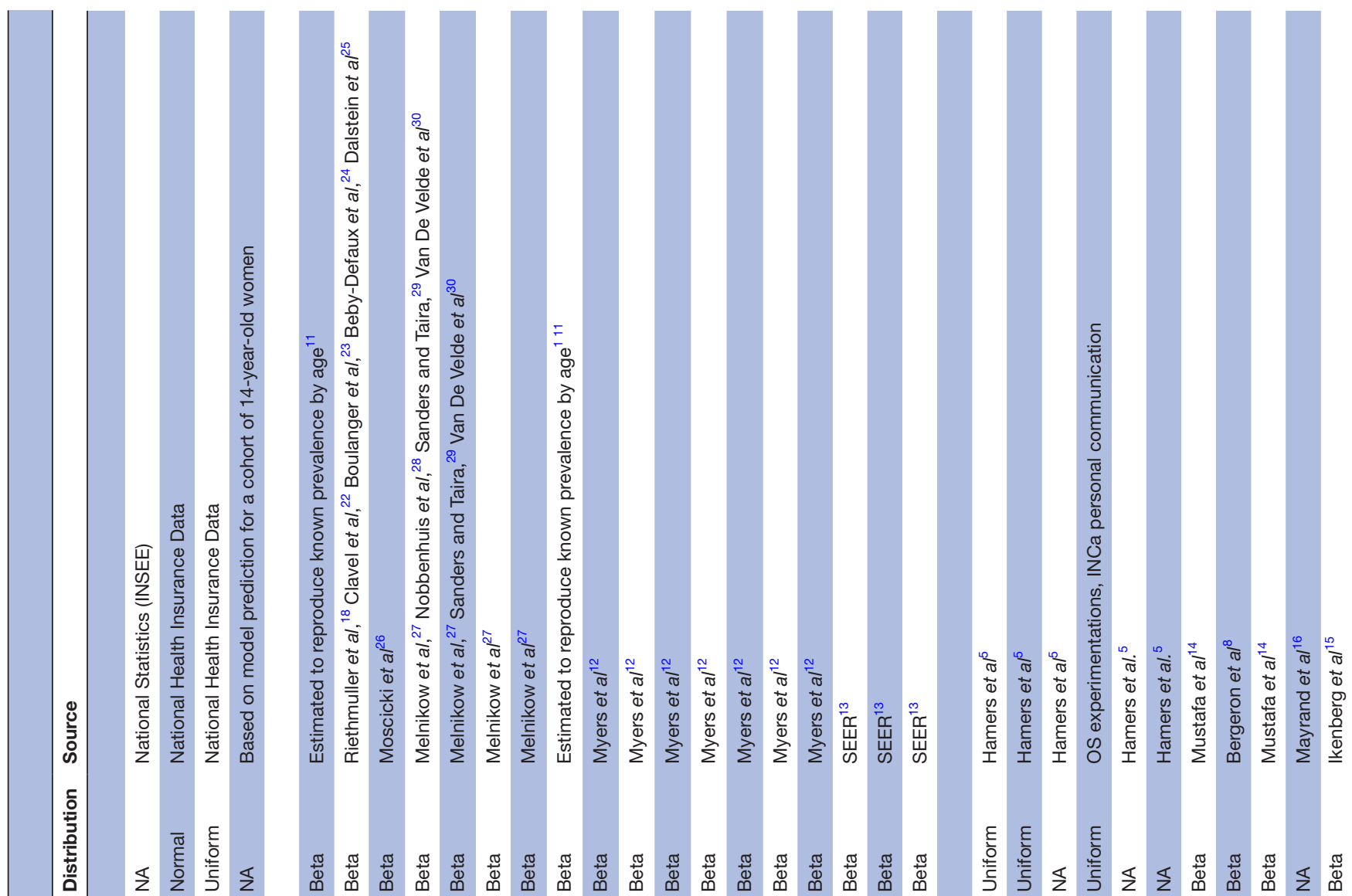

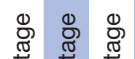
के

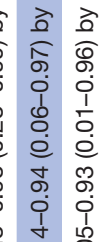

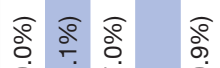

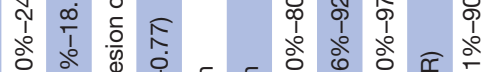
定

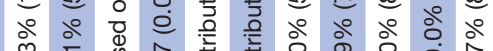

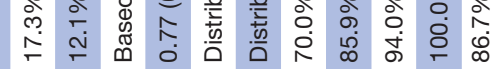

\section{宽:}

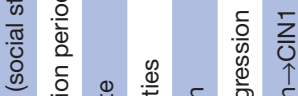

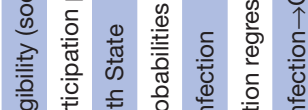

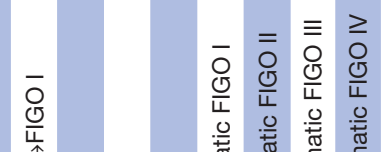

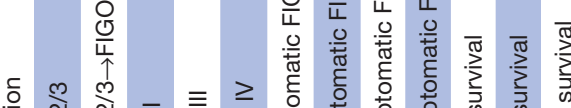

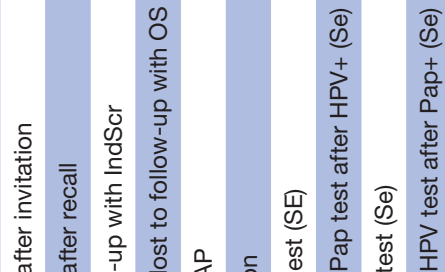

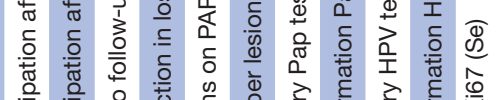

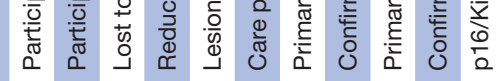




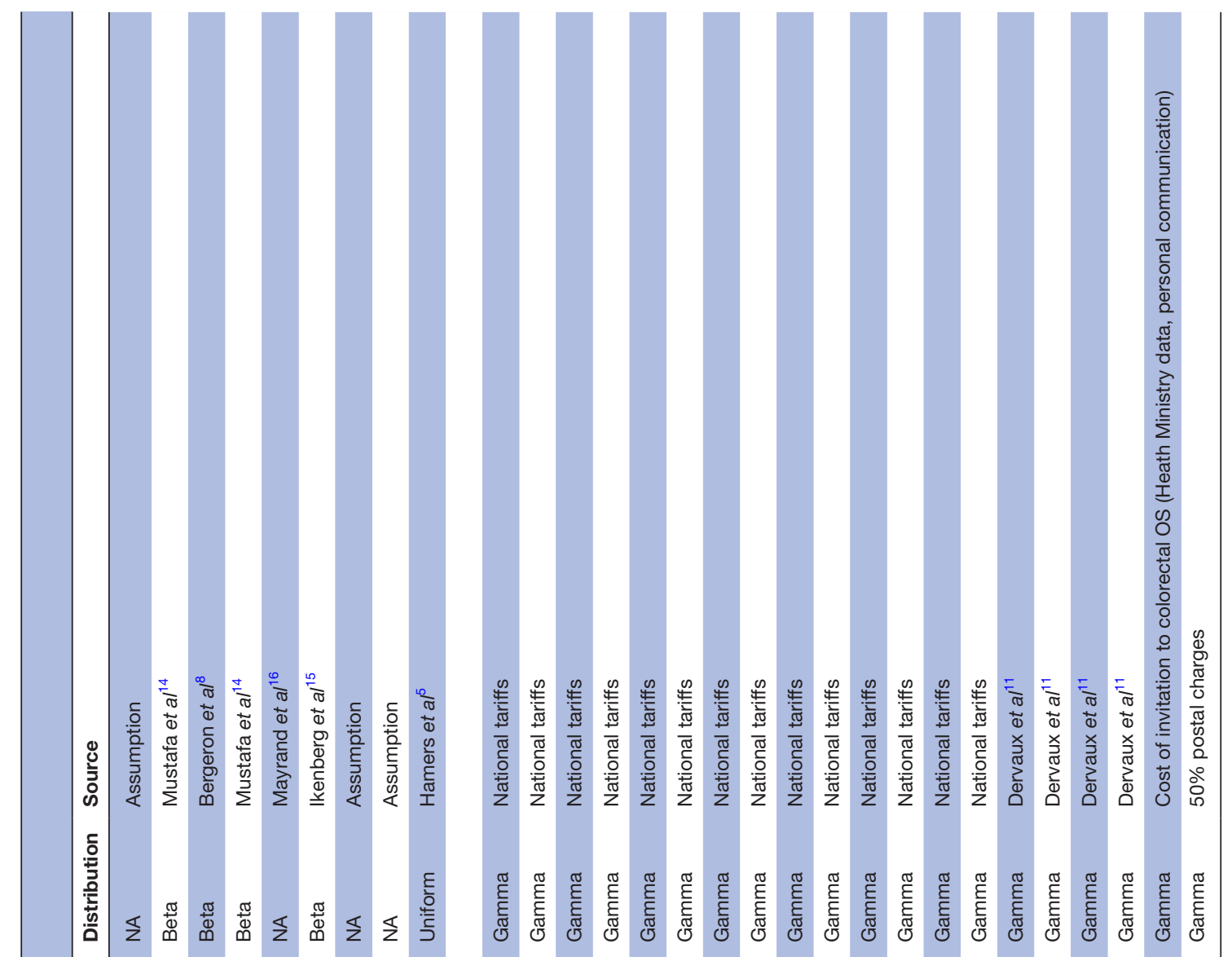

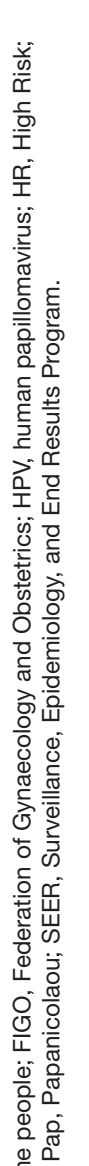

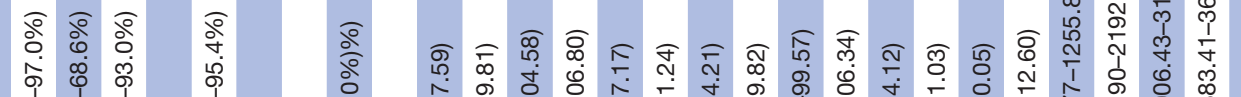

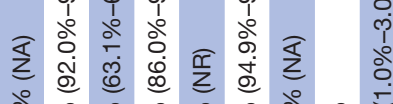

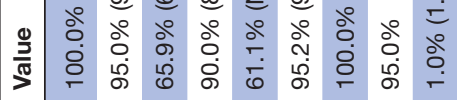

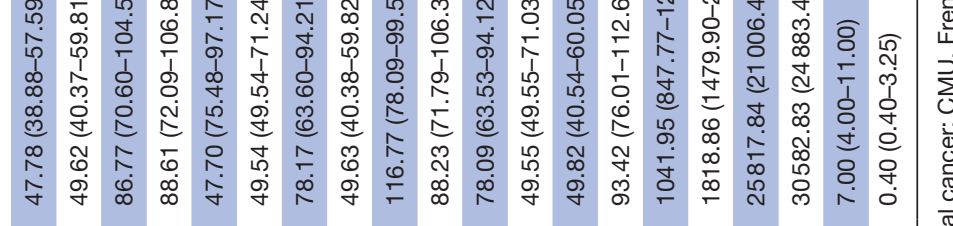

品

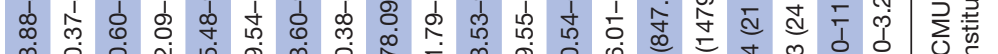

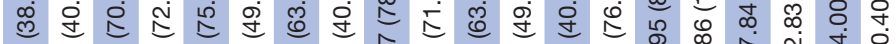

离离高

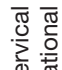

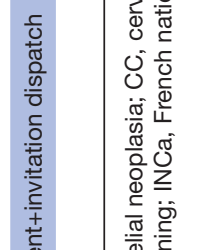

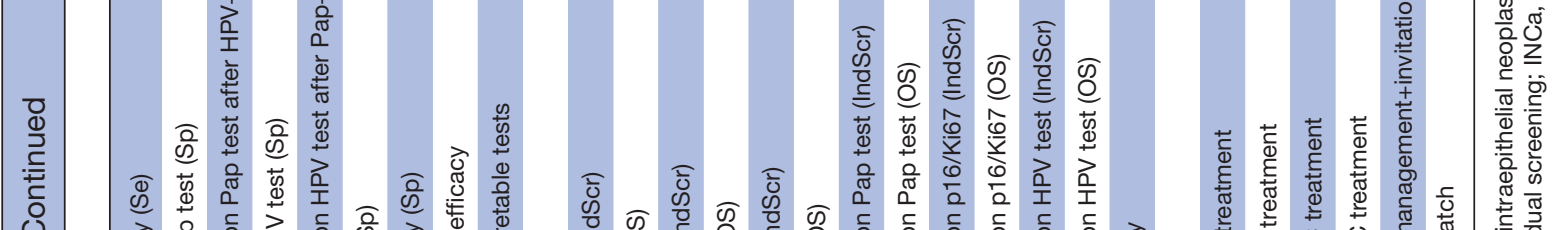


Table 3 Undiscounted results

\begin{tabular}{|c|c|c|c|c|c|c|}
\hline \multirow[b]{2}{*}{ Scenario } & \multicolumn{2}{|c|}{ Outcomes } & \multicolumn{4}{|c|}{ Costs (€) per woman } \\
\hline & Cancer & Cancer mortality & OS organisation & Screening & CC care and conisations & Total \\
\hline IndScr only* & 34 & 13 & 0 & 294.2 & 30.9 & 325.0 \\
\hline Pap/p16Ki67 & $-16.6 \%$ & $-15.9 \%$ & +19.57 & +18.46 & -4.57 & +33.46 \\
\hline HPV/Pap-3y & $-21.1 \%$ & $-22.4 \%$ & +15.16 & +99.87 & -7.31 & +107.73 \\
\hline HPV/Pap-10y & $-8.0 \%$ & $-13.6 \%$ & +14.94 & -14.42 & -0.48 & -134.04 \\
\hline HPV/p16Ki67-5y & $-22.9 \%$ & $-25.8 \%$ & +15.10 & +1.57 & -0.81 & +8.55 \\
\hline HPV/p16Ki67-10y & $-11.9 \%$ & $-17.0 \%$ & +14.93 & -129.7 & -5.87 & -120.63 \\
\hline p16Ki67/p16Ki67 & $-24.3 \%$ & $-24.4 \%$ & +19.57 & +233.30 & -6.87 & +246.00 \\
\hline
\end{tabular}

${ }^{*}$ Reference for other scenarios. Cumulated incidence and mortality for 10000 women eligible for OS on a lifetime horizon.

CC, cervical cancer; HPV, human papillomavirus; IndScr, individual screening; OS, organised screening; Pap, Papanicolaou.

$(\mathrm{RR}=0.88)$, observed lesions on Pap smear and associated care were all based on observational data from French OS experimentations. ${ }^{1}$

The sensitivity and specificity of screening tests were based on clinical studies for detecting CIN2/3 lesions and took into account the test sequence (ie, HPV after Pap or primary HPV). ${ }^{814-16}$ One per cent of Pap tests were non-interpretable, which led to a retest. ${ }^{17}$ Colposcopy was assumed to have $100 \%$ sensitivity and specificity. A $95 \%$ efficacy was considered for conisation.

The model estimated OS cost and direct medical costs from a collective, 'all payers' perspective, as recommended for France. ${ }^{18}$ The OS costs covered invitations and recalls, as well as database management, tracking of women's participation and FU management. Cost data for consultations and medical care were based on national tariffs. No extra consultation costs were added, as it was considered that IndScr participants did so during a routine consultation. The HPV-analysis tariff was decreased by $60 \%$ in strategies with primary HPV testing, assuming a substantial cost reduction in cases of an adoption of HPV testing-based OS. This assumption was validated by health insurance and health ministry representatives. According to public-health law, no extra co-payment is applied to OS participants. Cancer states were associated with costs accounting for care and FU by FIGO stage ${ }^{11}$ when entering the corresponding diagnosed state. All costs were updated to 2016, using the national consumer price index for healthcare goods and services.

\section{Validation}

The model results were compared with observed epidemiological data for validation. The model faithfully reproduces cancer incidence and CC mortality in France. ${ }^{11}$ Results of the model validation are available in the online supplementary file 5 .

\section{Cost-effectiveness analyses}

Incremental cost-effectiveness ratios (ICERs) were calculated for the life expectancy. Costs and survival were discounted at $4 \%$ per year, according to French guidelines for cost-effectiveness studies. ${ }^{17}$

Several alternative scenarios were tested, including not applying the efficacy of OS on LtFU rate, not considering a reduction in HPV cost and assuming a $60 \%$ reduction in $\mathrm{p} 16 / \mathrm{Ki} 67$ cost.

The robustness of the model was tested using deterministic sensitivity analysis (DSA). In the DSA, all of the parameters were tested at their CIs (or at $\pm 20 \%$ of the baseline value when the CIs were not available).

\section{RESULTS}

Compared with the current situation, invitation and recall of non-participant women led to an increase from $61.9 \%$ to $65.5 \%$ in the 4 -year participation rate. Every strategy that was tested was associated with a reduction in cancer incidence/mortality, ranging from $-14.2 \% /-13.5 \%$ for the Pap/Pap strategy to $-22.9 \% /-25.8 \%$ for the HPV/p16Ki67-5y strategy. The undiscounted results are presented in table 3.

The average undiscounted cost of screening for the modelled population over a lifetime was €325 per eligible woman, most of which was imputable to screening (€294). Strategies based on HPV testing with 5-year and 10 -year frequencies were cost saving $(-€ 22$ and $-€ 134$ per woman, respectively), despite the additional cost of OS $(€ 15)$. Other strategies were responsible for extra costs, ranging from $€ 29$ to $€ 33$ for Pap-based screening to $€ 108$ for HPV/Pap-3y and €246 for p16Ki67/p16Ki67.

Although it was the cheapest strategy ( $€ 191$ per eligible woman), HPV/Pap-10y was the strategy with the smallest cancer reduction $(-11.9 \%)$, as opposed to p16Ki67/ p16Ki67, which led to a 25\% reduction in CC while being the most expensive strategy ( $€ 571$ per eligible woman). Figure 2 presents the mean cost per woman and cancer reduction rate for each strategy.

Discounted survival is consistent with CC incidence and mortality (table 4). Compared with the current 
Table 4 Discounted results

\begin{tabular}{lllll}
\hline Scenario & Survival (LY) & Total cost (K€) & ICER (€/LY) versus current & Frontier \\
\hline IndScr only* & 19.4 & 122.6 & Reference & Dominated \\
Pap/Pap-HPV & +10.04 & +22.3 & 22234 & Dominated \\
Pap/p16Ki67 & +11.68 & +25.5 & 21918 & Dominated \\
HPV/Pap-3y & +15.93 & +55.8 & 35095 & Dominated \\
HPV/Pap-5y & +15.89 & -13.3 & Dominant & Ext. Dominated \\
HPV/Pap-10y & +10.51 & -73.4 & Dominant & Reference \\
HPV/p16Ki67-5y & +18.13 & +3.79 & 2091 & 101389 \\
HPV/p16Ki67-10y & +13.00 & -64.6 & Dominant & 35846 \\
p16Ki67/p16Ki67 & +18.37 & +160.7 & 87546 & 6592441 \\
\hline
\end{tabular}

${ }^{*}$ Reference for other scenarios. Extra survival per 10000 women eligible for OS on a lifetime horizon.

HPV, human papillomavirus; ICER, incremental cost-effectiveness ratio; IndScr, individual screening; LY, life years; OS, organised screening;

Pap, Papanicolaou.

situation (19.4 life years (LY) survival), OS strategies led to an increase in survival, ranging from 10 years per 10000 women for the Pap/Pap and HPV/Pap-10y strategies to 18 years per 10000 women for the $\mathrm{HPV} / \mathrm{p} 16 \mathrm{Ki} 67$ and p16Ki67/p16Ki67 strategies. Discounted extra costs per 10000 eligible women ranged from $€ 38000$ (HPV/ Pap-5y) to $€ 1608000$ (p16Ki67/p16Ki67). HPV/ Pap-5y and HPV/Pap-10y remained cost saving after discounting. Hence, these strategies were more effective and more cost saving than Pap-based strategies, including the current situation and were the dominant OS strategies. HPV/p16Ki67-5y and p16Ki67/p16Ki67 were more effective than HPV/Pap-5y and HPV/Pap-10y with ICERs of $€ 101391$ and $€ 6541250$ per LY, respectively. HPV/ Pap-3y was as effective as HPV/Pap-5y but less effective than HPV/p16Ki67-5y while generating much more expenses.
Regardless of the modality, implementing an OS programme for cervical cancer in France led to an overall improvement in the $\mathrm{CC}$ screening rate and a reduction in CC incidence and mortality. Reducing LtFU rates and improving screening rates with invitations/recall as in the Pap/Pap scenario results in an ICER of €22231 per LY and an average extra survival of $10 \mathrm{LY}$ per 10000 eligible women.

Switching primary screening from the Pap test to HPV testing led to similar LY gains with a 10-year screening frequency; yet, the 5-year frequency led to a longer survival (15.89 vs $10.51 \mathrm{LY}$ per 10000 eligible women). Furthermore, reducing the frequency of primary testing was cost saving, even at the current cost of HPV testing. Despite the longer interval between the two screening tests, HPV-based strategies remained effective because of their superior sensitivity compared with the Pap test.

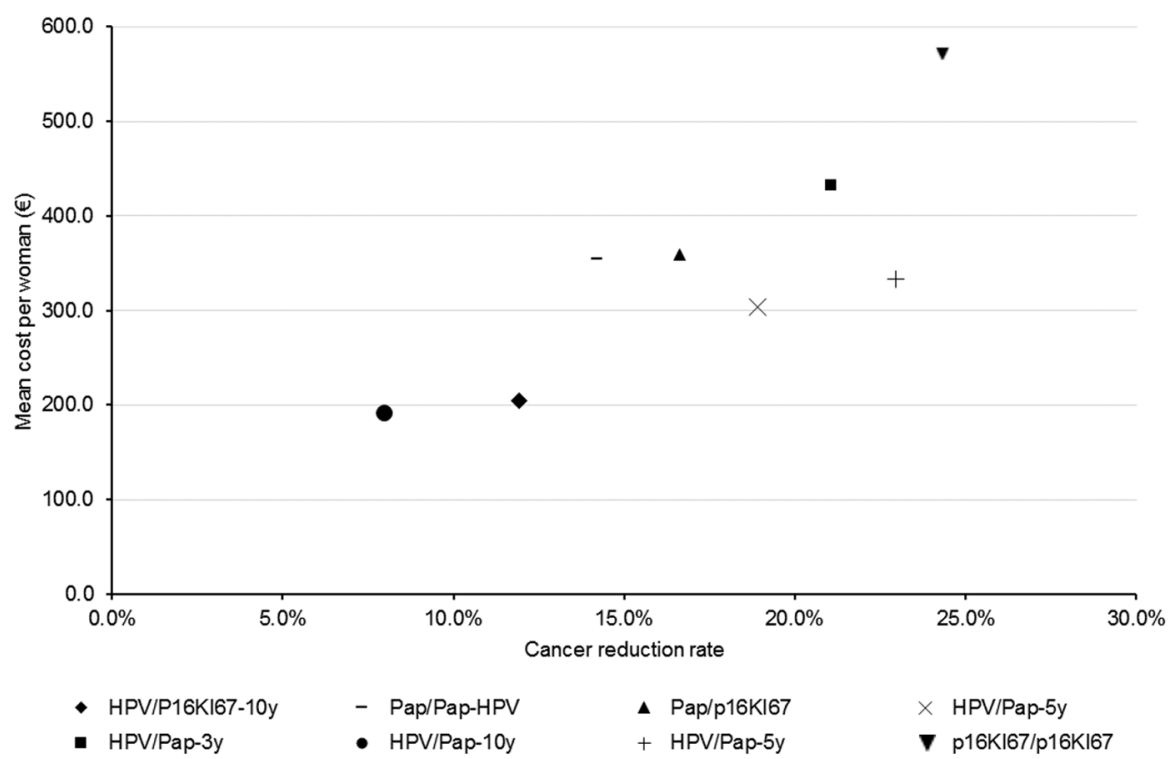

Figure 2 Results of organised screening strategies assessed on cancer reduction rate and associated mean cost. HPV, human papillomavirus; Pap, Papanicolaou. 


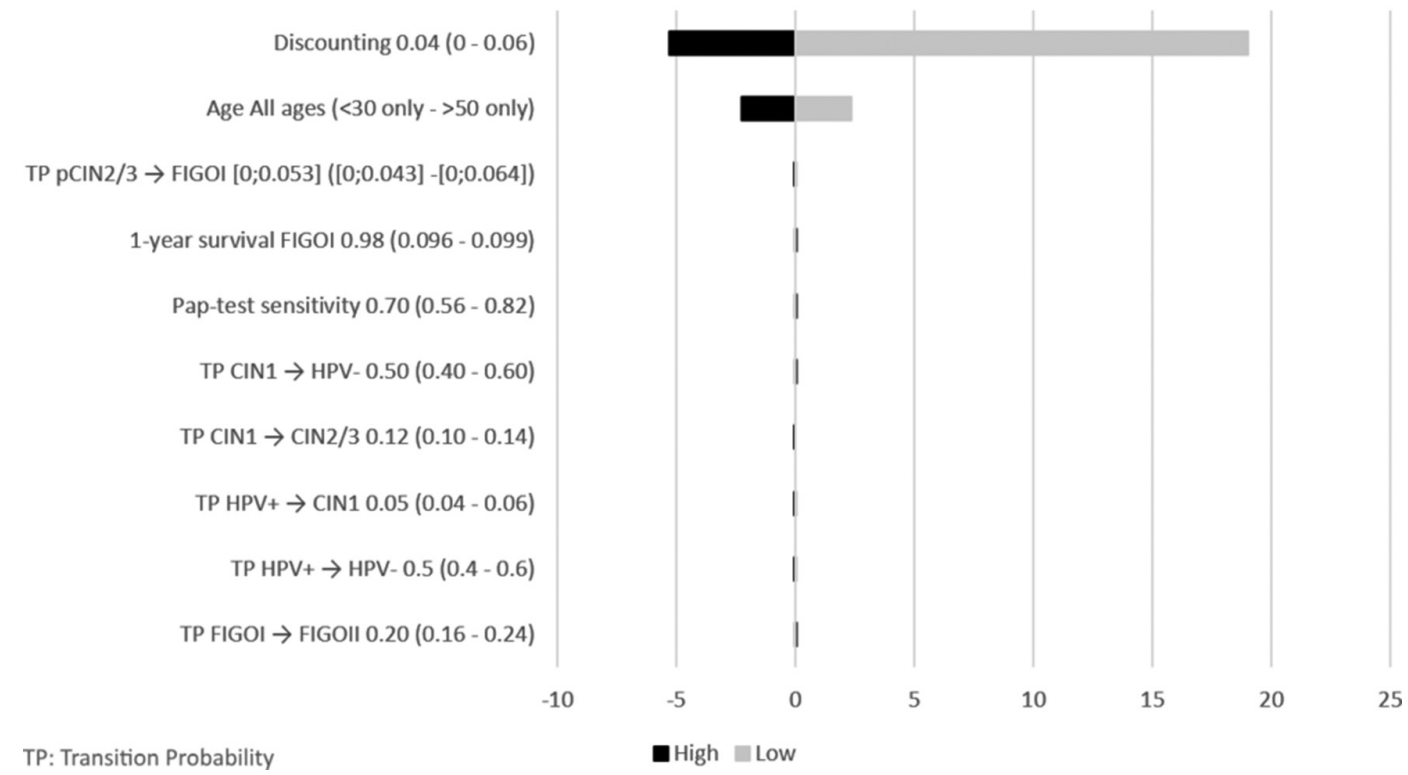

Figure 3 Tornado diagram of deterministic sensitivity analysis and scenario analyses with the biggest impact on survival for HPV/Pap-10y versus current situation. HPV, human papillomavirus; Pap, Papanicolaou.

The very good sensitivity/specificity of $\mathrm{p} 16 / \mathrm{Ki} 67$ double staining used as a primary screening test led to significant survival gains compared with the current situation and HPV testing $(+18.37$ and +2.48 per 10000 eligible women, respectively). However, its high cost made it inefficient, with an ICER of $€ 6592$ 441/LY.

Switching the Pap test with p16/Ki67 double staining in the confirmation of positive Pap and HPV primary tests increased efficacy and led to moderate additional costs. The confirmation of HPV tests every 10 years increased the survival from +10.51 to $+13.0 \mathrm{LY}$ and the costs from $-€ 734000$ to $-€ 646000$ per 10000 eligible women. Thus, the HPV/p16Ki67-10y scenario was associated with an ICER of $€ 35846 / \mathrm{LY}$. The cost-utility results do not lead to different conclusions. A cost-utility analysis was performed by applying specific health utilities to the health states and utility decrements to non-cancerous and cancerous states. Its results and the utility values used are available in the online supplementary file 6 .

\section{Sensitivity and scenario analyses}

Deterministic sensitivity analysis and scenario analyses for HPV/Pap-10y versus the current situation for LY and costs are shown in figures 3 and 4 , respectively.

The parameters with the biggest impact were the cost of testing (HPV and Pap) and OS effect on LtFU rate after a positive result. However, HPV/Pap-10y systematically remained the most cost-effective alternative. The mean

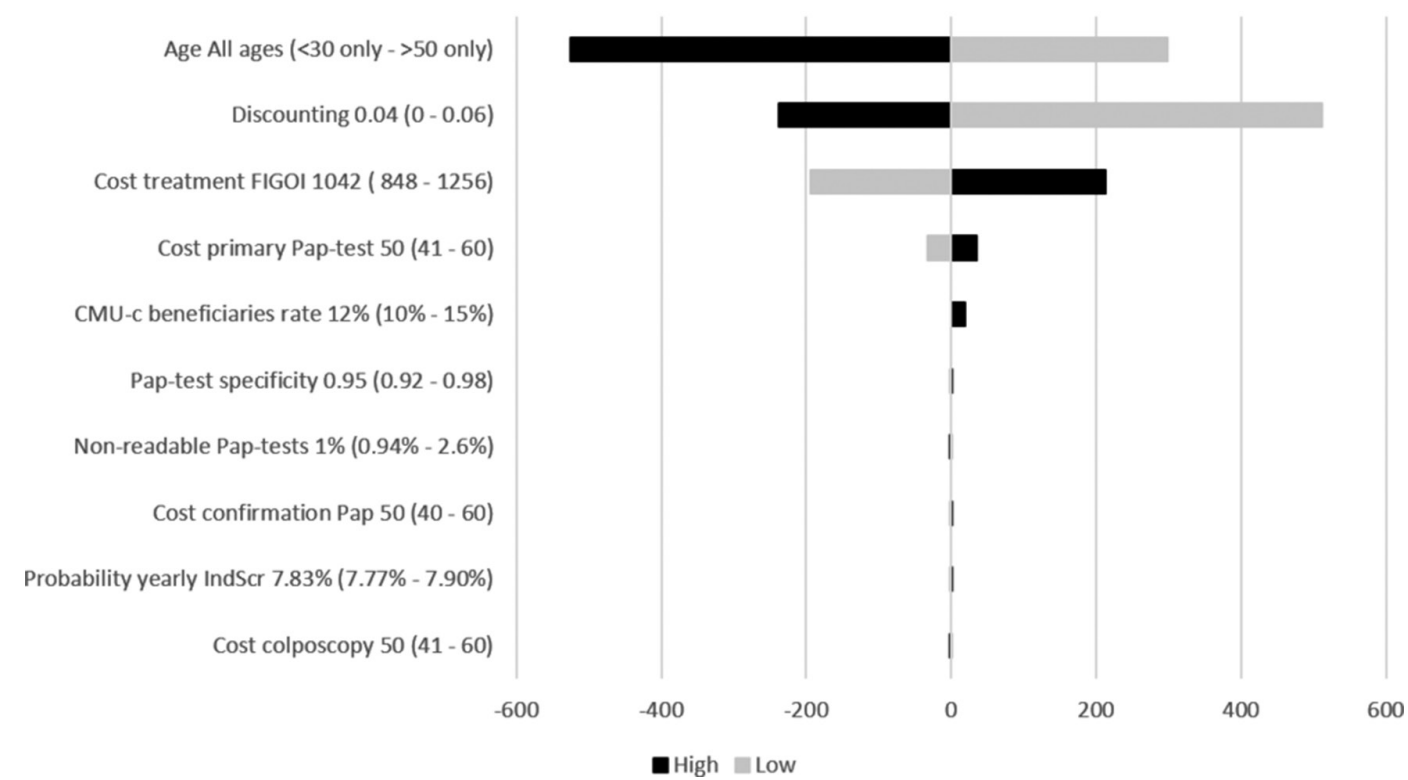

Figure 4 Tornado diagram of deterministic sensitivity analysis and scenario analyses with the biggest impact on costs for HPV/Pap-10y versus current situation. HPV, human papillomavirus; Pap, Papanicolaou 
age of the cohort impacted results drastically, despite HPV screening being less beneficial in women under 30 years old and over 50 years old than in the rest of the eligible population. Vaccination rates up to $80 \%$ had a negligible impact. Similar results were seen for HPV/Pap-5y and HPV/p16Ki67-5y scenarios. Not taking into account, the effect of OS on LtFU rate did not change the conclusion, although it significantly reduced the LY gains compared with the IndScr only. Similarly, not considering a reduction in the cost of the HPV test led to similar conclusions: HPV/Pap-10y and HPV/Pap-5y remained less costly than the alternative strategies. Finally, a $60 \%$ reduction in p16/ Ki67 cost led to a decreased total cost of $€ 41.05$ ( $-75 \%)$ for the p16Ki67/p16Ki67 scenario.

\section{DISCUSSION}

Using a validated microsimulation model that allows for the fine modelling of screening modalities, we showed that the OS programme for cervical cancer in France leads to a reduction of CC incidence and mortality. HPV-based screening with 5-year or 10-year frequencies would be cost saving, and other modalities would generate extra costs ranging between $€ 37.9$ and $€ 1607$ per woman.

Most model inputs were based on observed 'real-life' data instead of simple screening guidelines. This allows for an accurate simulation of women's screening behaviour by considering that many women do not comply with the recommended screening frequency and that older women tend to drop out of screening. ${ }^{3}$ This also allowed for the implementation of current professional practices that significantly differ from recommended screening algorithms: in the current IndScr only situation, after a positive Pap test, not all women proceed to confirmation (Pap or HPV test), as some directly undergo colposcopy or conisation, depending on the identified lesion with a significant impact on IndScr efficiency. Finally, the model incorporates $\mathrm{LtFU}$ rates, which proved to be a key factor in OS efficacy, particularly when screening frequency was superior to 5 years. ${ }^{5}$

The model's main limitations stem from the estimation of the transition probabilities (TP). An initial literature review showed important variations between sources with some TP being not available. Additionally, the identified TP were not precise enough given the low incidence of lesions in the general population of women ( 1 in 10 000). Thus, we favoured sources that had previously been used in French models to allow comparability with previously published results. ${ }^{10} 12$ Additionally, the model was calibrated on available prevalence data in France and externally validated. Furthermore, the sensitivity analyses showed that, despite the uncertainty, TP variations had a limited impact on the results, which reinforces our confidence in the estimations. Finally, our results are comparable to previously published European studies: Accetta et al have found that an HPV test every 5 years is more effective and less costly than triennial Pap tests in Italy. ${ }^{19}$ The decreased efficiency of CC screening based on the
HPV test at lower frequencies was shown by Berkhof et al in the Netherlands. ${ }^{20}$ In Norway, Burger et al found results comparable to ours for the Pap/Pap strategy. ${ }^{21}$

In our analysis, HPV/Pap-10y was the most efficient strategy, with HPV/p16Ki67-10y being a more cost-effective alternative. However, the final modality choice for OS implementation will need to consider several factors. First, the HPV/Pap-10y strategy, although the most efficient, is the less effective strategy in terms of cancer incidence and prevalence reduction, conflicting with the primary aim of the Cancer Plan to further reduce the CC burden in France ${ }^{4}$ and thus making the HPV/Pap-5y a more suitable, cost-saving modality. Second, current screening behaviours in France result in over-participation, with numerous women performing Pap tests more often than is recommended. This phenomenon is likely to be related to the yearly recommended consultation with a gynaecologist. Our results showed that going from a 5-year frequency to a 3-year frequency implies a huge increase in screening cost (from $-€ 133000$ to $+€ 558000$ per 10000 eligible women) for a very small increase in survival (from 15.89 to 15.93$)$. Indeed, HPV testing is sensitive, but it has a low specificity and cervical lesion evolution is slow, with most lesions regressing spontaneously. Women's over-participation will thus be a challenge in the case of HPV-based OS implementation. This should be addressed beforehand, or these apparent efficient strategies would be poorly efficient, leading to frequent false-positive results and related unnecessary and potentially harmful testing. Third, HPV testing is not recommended in women under 35 years of age, which would require a complex double screening system. Finally, the current screening organisation in France is based on the Pap test, which implies a different infrastructure. Switching to HPV would require the negotiation of HPV-test tariffs, the development of a quality assurance protocol to ensure a sensitivity that is consistent with those found during clinical studies, as well as the development of the required infrastructure and equipment. Thus, although primary HPV testing produces results with a better efficiency, many challenges will need to be addressed before its implementation. In the meantime, switching to a Pap test-based OS remains an acceptable alternative and could lead the way to HPV testing deployment.

As for $\mathrm{p} 16 / \mathrm{Ki} 67$ double staining, our results show that it would be an efficient confirmation test or primary test with negotiated tariffs. However, the sensitivity and specificity of the test were based on a single study with centralised reading. Additional studies in different French settings would be required to confirm that the results are reproducible before generalisation.

Lastly, we do not present our results relatively to a willingness-to-pay threshold. This choice results from the fact that no cost-effectiveness threshold is relevant in France, since the national agency in charge of health technology assessment, including pharmacoeconomic evaluation (HAS) does not wish cost-effectiveness results to be compared with a threshold. Indeed, cost-effectiveness 
analyses are not used as a resource allocation tool for health technologies in France. Furthermore, since implementation of CC OS was decided, we did not aim to assess whether and how OS was efficient, but to determine which screening modality was the most efficient, keeping in mind practical issues. We feel that this choice is further reinforced by our results that confirm the legislator's decision to implement OS. In summary, this modelling study enabled the INCa to provide robust information to support a public decision on both efficient intermediate modalities for implementation of the CC OS programme and also on optimal screening strategies in a longer term and to anticipate the integration of promising technological innovations.

Acknowledgements The authors acknowledge the members of the Scientific Committee for study for their critical review of the methodological choices, discussion of the results and conclusions of this medico-economic evaluation study: Jean Jacques Baldauf (Centre hospitalier universitaire de Strasbourg), Anne Sophie Banaszuk (Structure de gestion du Maine et Loire), Nathalie Beltzer (Santé Publique France), Mohamed-Béchir Ben Hadj Yahia (Centre hospitalier régional universitaire de Lille), Julia Bonastre (Institut Gustave Roussy), Véronique Dalstein (Centre hospitalier universitaire Reims), Marie Flori (Université de Lyon 1), Julie Gaillot (Institut National du Cancer), Chrystelle Gastaldi-Ménager (Caisse nationale d'assurance maladie des travailleurs salariés), Ken Haguenoer (Centre hospitalier régional universitaire de Tours), Françoise Hamers (Santé Publique France), Guy Launoy (Centre hospitalier universitaire de Caen, Inserm), Patricia Lucidarme (Collège national des sages-femmes), Emmanuel Ricard (Ligue Nationale contre le cancer), Jean-Paul Romarin (Agence régionale de santé du Languedoc-Roussillon Midi-Pyrénées), Catherine Rumeau-Pichon (Haute Autorité de Santé), Emmanuelle Salines (Ministère de la Santé), Nadia Thomas (Structure de gestion de Guyane), Alain Trugeon (Observatoire régional de santé de Picardie), Hélène Vandewalle (Institut National du Cancer), Anne Sophie Woronoff (Registre des cancers du Doubs), Laura Zanetti (Haute Autorité de Santé).

Contributors All authors participated in the study. SB, MM and HL participated in model development, data analysis and drafting of the manuscript. SB and FDB made critical review of the manuscript and approved its final version.

Funding This work was entirely funded by the National Cancer Institute.

Competing interests None declared.

Provenance and peer review Not commissioned; externally peer reviewed.

Data sharing statement No additional data available.

Open Access This is an Open Access article distributed in accordance with the Creative Commons Attribution Non Commercial (CC BY-NC 4.0) license, which permits others to distribute, remix, adapt, build upon this work non-commercially, and license their derivative works on different terms, provided the original work is properly cited and the use is non-commercial. See: http://creativecommons.org/ licenses/by-nc/4.0/

(c) Article author(s) (or their employer(s) unless otherwise stated in the text of the article) 2017. All rights reserved. No commercial use is permitted unless otherwise expressly granted.

\section{REFERENCES}

1. Santé HAde. État des lieux et recommandations pour le dépistage du cancer du col de l'utérus en France. 2010. http://www.has-sante.fr/ portail/jcms/c_1009772/fr/etat-des-lieux-et-recommandations-pourle-depistage-du-cancer-du-col-de-l-uterus-en-France

2. Monnereau A, Remontet L M, et al. Estimation nationale de l'incidence des cancers en France entre 1980 et 2012. Etude à partir des registres des cancers du réseau Francim - Partie 1 : tumeurs solides. 2013. http://invs.santepubliquefrance.fr/Publications-etoutils/Rapports-et-syntheses/Maladies-chroniques-et-traumatismes/ 2013/Estimation-nationale-de-I-incidence-des-cancers-en-Franceentre-1980-et-2012

3. Institut National du Cancer. Généralisation du dépistage du cancer du col de l'utérus /Étude médico-économique /Phase 1. 2015.
http://www.e-cancer.fr/Expertises-et-publications/Catalogue-despublications/Synthese-Generalisation-du-depistage-du-cancer-ducol-de-l-uterus-etude-medico-economique-Phase-1

4. Ministère des Affaires sociales et de la santéMinistère de l'Enseignement supérieur et de la recherche. Plan Cancer 20142019, Guérir et prévenir les cancers : donnons les mêmes chances à tous, partout en France. 2014. http://www.e-cancer.fr/Plan-cancer/ Plan-cancer-2014-2019-priorites-et-objectifs

5. Hamers FF, Duport N, Beltzer N. Population-based organized cervical cancer screening pilot program in France. Eur J Cancer Prev 2017:1.

6. Haguenoer K, Sengchanh S, Gaudy-Graffin C, et al. Vaginal selfsampling is a cost-effective way to increase participation in a cervical cancer screening programme: a randomised trial. $\mathrm{Br} \mathrm{J}$ Cancer 2014;111:2187-96.

7. Dalstein V, Charlier B, Botokeky J, et al. Évaluation intermédiaire de START -HPV, programme pilote de dépistage primaire par test HPV des lésions précancéreuses et cancéreuses du col utérin dans le département des Ardennes, France. Bull Epidémiol Hebd 2014;15:254-9.

8. Bergeron $\mathrm{C}$, Ikenberg $\mathrm{H}$, Sideri $\mathrm{M}$, et al. Prospective evaluation of p16/Ki-67 dual-stained cytology for managing women with abnormal Papanicolaou cytology: PALMS study results. Cancer Cytopathol 2015;123:373-81.

9. Demarteau N, Detournay B, Tehard B, et al. A generally applicable cost-effectiveness model for the evaluation of vaccines against cervical cancer. Int J Public Health 2011;56:153-62.

10. Binder-Foucard F, Belot $A$, Delafosse $P$, et al. Estimation nationale de l'incidence et de la mortalité par cancer en France entre 1980 et 2005. Etude à partir des registres des cancers du réseau Francim - Partie 1 : tumeurs solides. 2008. 2008. http://invs. santepubliquefrance.fr/Publications-et-outils/Rapports-et-syntheses/ Maladies-chroniques-et-traumatismes/2013/Estimation-nationalede--incidence-et-de-la-mortalite-par-cancer-en-France-entre-1980et-2012

11. Dervaux B, Lenne X, Lévy-Bruhl $D$, et al. Modélisation médicoéconomique de l'impact de l'organisation du dépistage du cancer du col utérin et de l'introduction de la vaccination contre les HPV dans le calendrier vaccinal. 2008. https://www.mesvaccins.net/textes/ rapport_modelisation_hpv.pdf

12. Myers ER, McCrory DC, Nanda K, et al. Mathematical model for the natural history of human papillomavirus infection and cervical carcinogenesis. Am J Epidemiol 2000;151:1158-71.

13. Wright JD, Chen L, Tergas Al, et al. Population-level trends in relative survival for cervical cancer. Am J Obstet Gynecol 2015;213:670. e1-7.

14. Mustafa RA, Santesso N, Khatib R, et al. Systematic reviews and meta-analyses of the accuracy of HPV tests, visual inspection with acetic acid, cytology, and colposcopy. Int J Gynaecol Obstet 2016;132:259-65.

15. Ikenberg $\mathrm{H}$, Bergeron $\mathrm{C}$, Schmidt $\mathrm{D}$, et al. Screening for cervical cancer precursors with $\mathrm{p} 16 / \mathrm{Ki}-67$ dual-stained cytology: results of the PALMS study. J Natl Cancer Inst 2013;105:1550-7.

16. Mayrand MH, Duarte-Franco E, Rodrigues I, et al. Human papillomavirus DNA versus Papanicolaou screening tests for cervical cancer. N Engl J Med 2007;357:1579-88.

17. Santé HAde. Choix méthodologiques pour l'évaluation médicoéconomique à la HAS. 2011.http://www.has-sante.fr/portail/upload/ docs/application/pdf/2011-11/guide_methodo_vf.pdf

18. Riethmuller D, Gay C, Bertrand X, et al. Genital human papillomavirus infection among women recruited for routine cervical cancer screening or for colposcopy determined by Hybrid Capture II and polymerase chain reaction. Diagn Mol Pathol 1999;8:157-64.

19. Accetta G, Biggeri A, Carreras G, et al. Is human papillomavirus screening preferable to current policies in vaccinated and unvaccinated women? A cost-effectiveness analysis. J Med Screen 2010;17:181-9.

20. Berkhof J, Coupé VM, Bogaards JA, et al. The health and economic effects of HPV DNA screening in The Netherlands. Int $J$ Cancer 2010;127:2147-58

21. Burger EA, Kim JJ. The value of improving failures within a cervical cancer screening program: an example from Norway. Int $J$ Cancer 2014;135:1931-9.

22. Clavel C, Masure M, Bory JP, et al. Human papillomavirus testing in primary screening for the detection of high-grade cervical lesions: a study of 7932 women. Br J Cancer 2001;84:1616-23.

23. Boulanger JC, Sevestre H, Bauville E, et al. [Epidemiology of HPV infection]. Gynecol Obstet Fertil 2004;32:218-23.

24. Beby-Defaux A, Bourgoin A, Ragot S, et al. Human papillomavirus infection of the cervix uteri in women attending a Health Examination Center of the French social security. J Med Virol 2004;73:262-8. 
25. Dalstein V, Riethmuller D, Sautière JL, et al. Detection of cervical precancer and cancer in a hospital population; benefits of testing for human papillomavirus. Eur J Cancer 2004;40:1225-32.

26. Moscicki AB, Hills N, Shiboski S, et al. Risks for incident human papillomavirus infection and low-grade squamous intraepithelial lesion development in young females. JAMA 2001;285:2995-3002.

27. Melnikow J, Nuovo J, Willan AR, et al. Natural history of cervical squamous intraepithelial lesions: a meta-analysis. Obstet Gynecol 1998;92:727-35
28. Nobbenhuis MA, Helmerhorst TJ, van den Brule AJ, et al Cytological regression and clearance of high-risk human papillomavirus in women with an abnormal cervical smear. Lancet 2001;358:1782-3.

29. Sanders GD, Taira AV. Cost-effectiveness of a potential vaccine for human papillomavirus. Emerg Infect Dis 2003;9:37-48.

30. Van de Velde N, Brisson M, Boily MC. Modeling human papillomavirus vaccine effectiveness: quantifying the impact of parameter uncertainty. Am J Epidemiol 2007;165:762-75. 